\title{
TITLE:
}

\section{Photostability of Monolayer Transition-Metal Dichalcogenides in Ambient Air and Acidic/Basic Aqueous Solutions}

\section{$\operatorname{AUTHOR}(\mathrm{S}):$}

Zhang, Wenjin; Matsuda, Kazunari; Miyauchi, Yuhei

\section{CITATION:}

Zhang, Wenjin ...[et al]. Photostability of Monolayer Transition-Metal Dichalcogenides in Ambient Air and Acidic/Basic Aqueous Solutions. ACS Omega 2019, 4(6): 10322-10327

\section{ISSUE DATE:}

2019-06-30

URL:

http://hdl.handle.net/2433/242866

\section{RIGHT:}

(C) 2019 American Chemical Society. This is an open access article published under a Creative Commons Attribution (CC-BY)License, which permits unrestricted use,

distribution and reproduction in any medium, provided the author and source are cited. 


\title{
Photostability of Monolayer Transition-Metal Dichalcogenides in Ambient Air and Acidic/Basic Aqueous Solutions
}

\author{
Wenjin Zhang, Kazunari Matsuda, ${ }^{\circledR}$ and Yuhei Miyauchi*io \\ Institute of Advanced Energy, Kyoto University, Uji, Kyoto 611-0011, Japan \\ Supporting Information
}

\begin{abstract}
We report on the photostability of monolayer (1L) transition-metal dichalcogenides (TMDCs) in air and in aqueous solutions, as probed using photoluminescence spectroscopy. 1L$\mathrm{WSe}_{2}$ was readily degraded under continuous irradiation of visible light in aqueous solutions, whereas $1 \mathrm{~L}-\mathrm{MoS}_{2}$ was relatively stable in both ambient air and aqueous solutions. The stability difference between these two materials was mainly ascribed to the oxidization reaction at the interface of 1L-TMDCs and the $\mathrm{O}_{2} / \mathrm{H}_{2} \mathrm{O}$ redox system induced by both band alignment and photogenerated holes. This interpretation was strongly supported by the observation of the lower degradation rate of $1 \mathrm{~L}-\mathrm{WSe}_{2}$ in the dark and in degassed water with a lower concentration of dissolved oxygen compared with the degradation rate of $1 \mathrm{~L}-\mathrm{WSe}_{2}$ in distilled water. Furthermore,

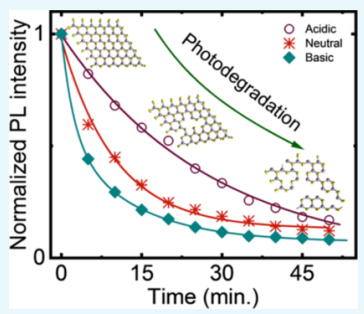
the degradation rate was also nearly proportional to the number of photogenerated carriers. The degradation rate under acidic conditions was smaller than that under the basic conditions. The results are attributed to the oxidation/reduction potential of $1 \mathrm{~L}-\mathrm{WSe}_{2}$ and to the dissolution reaction of degraded species, both of which are strongly $\mathrm{pH}$-dependent.
\end{abstract}

\section{INTRODUCTION}

Monolayers (1L) of transition-metal dichalcogenides (TMDCs) $\mathrm{MX}_{2}$, where $\mathrm{M}$ is a transition metal (typically Mo or $\mathrm{W}$ ) and $\mathrm{X}$ is a chalcogen (typically $\mathrm{S}, \mathrm{Se}$, or Te), have emerged as new direct-gap semiconductors with truly twodimensional structures. Because of the striking change in their electronic structures from indirect $(>2 \mathrm{~L})$ to direct band gap (1L) depending on their number of layers, ${ }^{1-3}$ 1L-TMDCs show interesting optical and electrical properties which have strong potential for use in future electronic and optoelectronic devices such as phototransistors, ${ }^{4-9}$ photodetectors, ${ }^{10-12}$ lightemitting devices, ${ }^{13,14}$ light modulators, ${ }^{15}$ and solar cells. ${ }^{16-18}$ Tunable photoluminescence (PL) has thus far been demonstrated using chemisorption and physisorption, ${ }^{19-22}$ covalent bonding, , $24^{24}$ and electrostatic methods. ${ }^{25,26}$ The electronic properties have also been studied using chemical doping and defect engineering. ${ }^{27-29}$ Additionally, the applications of 1LTMDCs as gas sensors or biosensors and photocatalysts for water splitting have been reported..$^{30-35}$ Electrochemical etching or oxidation reactions of atomically thin TMDCs under photoirradiation ${ }^{36,37}$ or ozone exposure ${ }^{38}$ conditions were also studied. These previous studies suggest that deep understanding of the influence of surface reactions on the physical properties and stability of 1L-TMDCs is important for realizing their application in various environments such as ambient air or wet conditions. However, knowledge of the stability of $1 \mathrm{~L}-\mathrm{TMDC}$ under such conditions is limited.

In this paper, we report on the stability of the most typical $1 \mathrm{~L}-\mathrm{TMDCs}-1 \mathrm{~L}-\mathrm{WSe} \mathrm{e}_{2}$ and $1 \mathrm{~L}-\mathrm{MoS}_{2}$ - in ambient air and acidic/basic aqueous solutions under visible-light irradiation. The PL intensities of $1 \mathrm{~L}-\mathrm{WSe}_{2}$ distinctly decreased in acidic/ basic aqueous solutions, whereas $\mathrm{MoS}_{2}$ was stable under the same conditions and its PL intensity showed a negligible decrease. The PL spectral shape did not show a remarkable change with increasing irradiation time for either $1 \mathrm{~L}-\mathrm{WSe}_{2}$ or $1 \mathrm{~L}-\mathrm{MoS}_{2}$, suggesting that the change in the PL intensity was not caused by photoinduced carrier doping but was mainly attributable to photoinduced degradation. We confirmed that the degradation rate was lower in the dark and in degassed water than in distilled water, suggesting that both light and dissolved oxygen are necessary for photodegradation reactions. Furthermore, $1 \mathrm{~L}-\mathrm{WSe}_{2}$ under acidic and neutral conditions showed a more moderate degradation rate than that under the basic condition. These results suggest that the electrochemical reaction with $1 \mathrm{~L}-\mathrm{TMDCs}$ and photogenerated holes in the presence of oxygen and water is the key mechanism of the degradation. This mechanism is effective for $1 \mathrm{~L}-\mathrm{WSe}_{2}$ and ineffective for $\mathrm{MoS}_{2}$ because of the difference in the relative alignment among the oxidation/reduction potentials of $\mathrm{O}_{2} /$ $\mathrm{H}_{2} \mathrm{O}$ and the levels of the valence and conduction bands of each $1 \mathrm{~L}-\mathrm{TMDC}$.

\section{RESULTS AND DISCUSSION}

Figure 1 shows the schematic of the optical system for the PL measurements in air or aqueous solutions. 1L-TMDCs prepared on a transparent glass substrate (Figure S1) were irradiated with visible wavelength light $(580 \mathrm{~nm})$ with a constant power density $\left(800 \mathrm{~W} \mathrm{~cm} \mathrm{~cm}^{-2}\right)$. The signal was recorded using a silicon charge-coupled device (CCD) detector equipped with a spectrometer (see the Experimental Methods section for details).

Received: April 15, 2019

Accepted: May 31, 2019

Published: June 13, 2019 


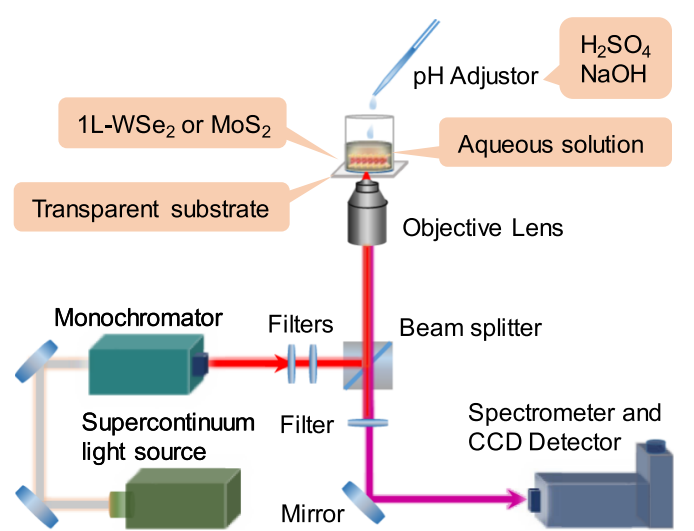

Figure 1. Schematic of the optical system for the PL measurement. White light from a supercontinuum light source is separated using a monochromator and optical filters, and only visible light with selected wavelength is focused on to the sample on a glass substrate. The samples were placed in air or in aqueous solution during the measurement.

Figure 2a,b shows the PL intensity and the normalized PL spectra of $1 \mathrm{~L}-\mathrm{WSe}_{2}$ under ambient air at different photo- (a)

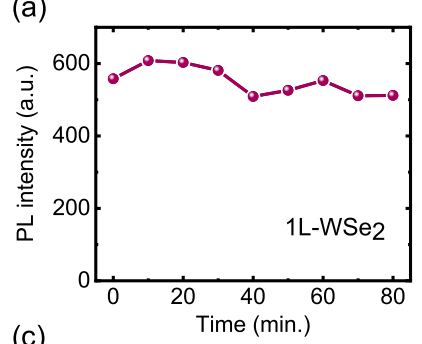

(c)

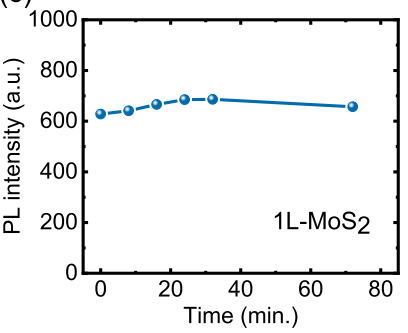

(b)

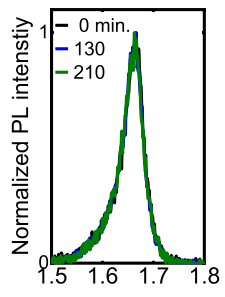

(d)

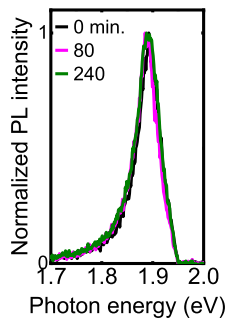

Figure 2. Time-dependent PL intensity $(\mathrm{a}, \mathrm{c})$ and normalized PL spectra $(b, d)$ in ambient air. $(a, b)$ are for $1 \mathrm{~L}-W_{S} e_{2}$ and $(c, d)$ are for $1 \mathrm{~L}-\mathrm{MoS}_{2}$.

irradiation times. As shown in Figure 2a, the PL intensity decreased slightly within 90 min under photoirradiation (580 $\mathrm{nm}, 800 \mathrm{~W} \mathrm{~cm}^{-2}$, which is in the linear region shown in Figure S2). By contrast, $1 \mathrm{~L}-\mathrm{MoS}_{2}$ showed a negligible change in PL intensity (Figure 2c). The normalized PL spectra show an undetectable shape change in the spectra of both $1 \mathrm{~L}-\mathrm{WSe}_{2}$ and $1 \mathrm{~L}-\mathrm{MoS}_{2}$ (Figure 2b,d). Because the optical absorption coefficients of $1 \mathrm{~L}-\mathrm{TMDCs}$ at $580 \mathrm{~nm}$ are similar $\left(1 \times 10^{5}\right.$ to $\left.2 \times 10^{5} \mathrm{~cm}^{-1}\right),{ }^{39,40}$ we expected similar numbers of electrons and holes to be photogenerated in these samples. Thus, these results imply that the stability of $1 \mathrm{~L}-\mathrm{WS} \mathrm{e}_{2}$ is slightly lower than that of $\mathrm{MoS}_{2}$ in ambient air under photoirradiation.

Because the properties of $1 \mathrm{~L}-\mathrm{TMDCs}$ are known to be strongly modified by electrochemical effects at the surfaces, ${ }^{22,41}$ we next studied their stabilities in water under light irradiation. Under these conditions, water molecules are adsorbed onto the surface and electrochemical effects because the oxygen/water redox couple becomes important. ${ }^{41}$ We first performed PL measurements with the 1L-TMDCs in distilled water and then with the $1 \mathrm{~L}-\mathrm{TMDCs}$ in acidic/basic solutions. As shown in Figure $3 a, c$, the PL intensity of $1 \mathrm{~L}-\mathrm{WSe}_{2}$ (Figure $3 \mathrm{a})$ in aqueous solution rapidly decreased with increasing irradiation time, whereas the PL intensity of $1 \mathrm{~L}-\mathrm{MoS}_{2}$ (Figure $3 \mathrm{c}$ ) was stable under all of the investigated conditions. The main decrease of the $1 \mathrm{~L}-\mathrm{WSe} \mathrm{S}_{2} \mathrm{PL}$ intensity under neutral (distilled water) and basic conditions occurred within the first 10-15 $\mathrm{min}$; the decrease then slowed, likely because of the increased concentration of the degradation products from $1 \mathrm{~L}$ $\mathrm{WSe}_{2}$. Under the acidic condition, as shown in Figure 3a, 1L$\mathrm{WSe}_{2}$ exhibited a relatively moderate degradation rate, in contrast to the fast degradation observed under other conditions. The original PL intensities decreased by approximately 15,40 , and $55 \%$ within $10 \mathrm{~min}$ in acidic $(\mathrm{pH}=2)$, neutral $(\mathrm{pH}=7)$, and basic $(\mathrm{pH}=12)$ conditions, respectively. The normalized PL spectra of $1 \mathrm{~L}-\mathrm{WSe}_{2}$ and $1 \mathrm{~L}-\mathrm{MoS}_{2}$ show almost no change of the spectral shape within the irradiation time as shown in Figure $3 \mathrm{~b}, \mathrm{~d}$. We also confirmed the photoinduced degradation of $1 \mathrm{~L}-\mathrm{WSe}_{2}$ in water directly by observing optical and PL images before and after the photoirradiation (Figure S3).

To elucidate the mechanism of the material-dependent degradation behavior and the differences among degradation rates of the materials in different environments, we consider $\mathrm{O}_{2} / \mathrm{H}_{2} \mathrm{O}$ redox couple and the electrochemical reaction at the interface between aqueous solutions and 1L-TMDCs. The effects of $\mathrm{O}_{2}$ and $\mathrm{H}_{2} \mathrm{O}$ molecules on the optical and electrical properties of the $1 \mathrm{~L}$-TMDCs were reported. ${ }^{22,41}$ The transfer of photogenerated electrons or holes may be a main factor related to the degradation or decomposition of the 1L-TMDCs in an $\mathrm{O}_{2} / \mathrm{H}_{2} \mathrm{O}$ system, depending on the band alignment of the semiconductors with the oxidation/reduction potentials of $\mathrm{O}_{2} /$ $\mathrm{H}_{2} \mathrm{O}$. In general, photogenerated holes $\left(\mathrm{h}^{+}\right)$can facilitate the oxidation of compound semiconductors $(\mathrm{AB})^{42}$ and destruct their original structures

$$
\mathrm{AB}+z \mathrm{~h}^{+}+\text {solv } \rightarrow \mathrm{A}(\text { oxidation })+\mathrm{B}^{z+} \bullet \text { solv }
$$

where $z$ represents the number of holes and solv is solvent. In the current study, A and B are the transition metal and chalcogen that compose the semiconductors, respectively.

The band alignment of $1 \mathrm{~L}-\mathrm{WS} \mathrm{e}_{2}$ relative to vacuum is shown in Figure 4a. The conduction-band minimum is near $-3.6 \mathrm{eV}$, which is much higher than the $\mathrm{H}^{+} / \mathrm{H}_{2}$ reduction potential (ca. $-4.83 \mathrm{eV}) .^{43-47}$ Thus, some of the photogenerated electrons can reduce $\mathrm{H}^{+}$to $\mathrm{H}_{2}$. This process can also occur for $1 \mathrm{~L}-\mathrm{MoS}_{2}$ (Figure 4b), which has a conduction-band minimum at approximately $-4.1 \mathrm{eV}^{47,48}$ however, the smaller energy difference from $\phi\left(\mathrm{H}^{+} / \mathrm{H}_{2}\right)$ results in a lower electron driving force/rate to reduce $\mathrm{H}^{+}$to $\mathrm{H}_{2}$ in comparison with $1 \mathrm{~L}-\mathrm{WSe}_{2}$, as reported in previous studies. ${ }^{49,50}$ The valance-band edge of $1 \mathrm{~L}$ $\mathrm{WSe}_{2}$ is at approximately $-5.2 \mathrm{eV},{ }^{47,51}$ which is higher than the $\mathrm{O}_{2} / \mathrm{H}_{2} \mathrm{O}$ potential (ca. $-5.66 \mathrm{eV}$ ). Photogenerated holes in $\mathrm{WSe}_{2}$ cannot totally recombine with the photogenerated electrons because some of the electrons have already been used to reduce $\mathrm{H}^{+}$to $\mathrm{H}_{2}$. Thus, the excess amount of holes degrades the $1 \mathrm{~L}-\mathrm{WSe}_{2}$ itself rather than oxygenating $\mathrm{H}_{2} \mathrm{O}^{42,52}$ that is energetically unfavorable. These processes are expressed in eqs 2 and $3^{36,53,54}$

$$
2 \mathrm{H}^{+}+2 \mathrm{e}^{-} \rightarrow \mathrm{H}_{2}
$$


(a)

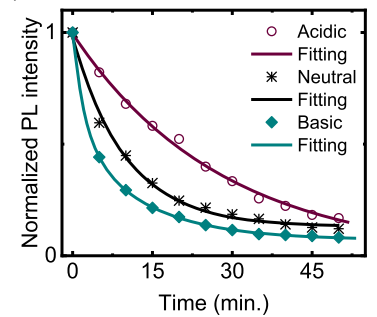

(c)

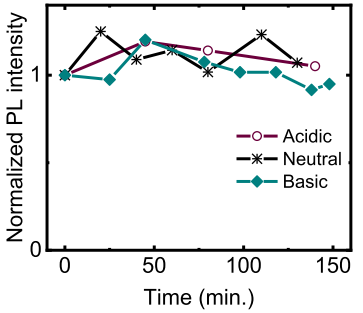

(b)

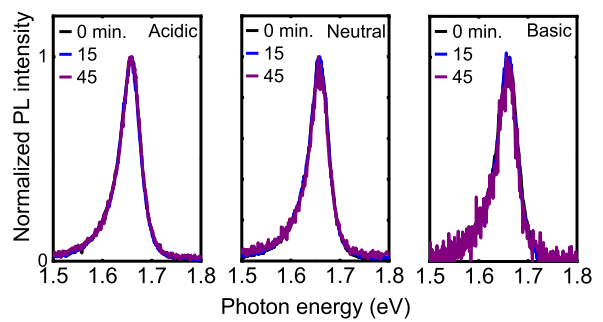

(d)

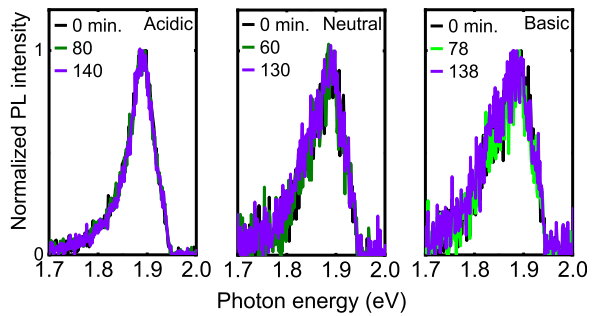

Figure 3. Time-dependent PL intensity and the spectral shape of $(\mathrm{a}, \mathrm{b}) 1 \mathrm{~L}-\mathrm{WS} \mathrm{e}_{2}$ and $(\mathrm{c}, \mathrm{d}) 1 \mathrm{~L}-\mathrm{MoS}_{2}$ under acidic, neutral, and basic conditions. The solid curves in (a) are the results of fitting using single- or double-exponential functions.

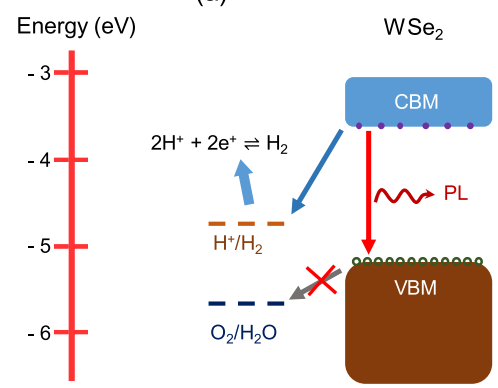

(a) (b)

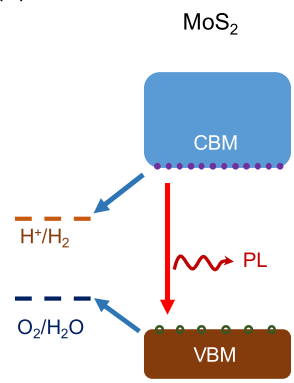

Figure 4. Band alignment of (a) $1 \mathrm{~L}-\mathrm{WSe}_{2}$ and (b) $1 \mathrm{~L}-\mathrm{MoS}_{2}$ with the water redox potential.

$$
\begin{aligned}
& 2 \mathrm{WSe}_{2}+8 \mathrm{~h}^{+}+8 \mathrm{H}_{2} \mathrm{O}+7 \mathrm{O}_{2} \\
& \rightleftharpoons 2 \mathrm{WO}_{3}+4 \mathrm{SeO}_{4}{ }^{2-}+16 \mathrm{H}^{+}
\end{aligned}
$$

For $1 \mathrm{~L}-\mathrm{MoS}_{2}$, the valence-band maximum is at approximately $-6.0 \mathrm{eV}$ which is lower than the potential of $\mathrm{O}_{2} / \mathrm{H}_{2} \mathrm{O}$ and some of the photogenerated holes may oxygenate $\mathrm{H}_{2} \mathrm{O}$ to $\mathrm{O}_{2}$. In this case, fewer excess holes remain and $\mathrm{MoS}_{2}$ itself is not likely to be degraded within a short time. Thus, we can explain the difference in the degradation behaviors between $1 \mathrm{~L}-\mathrm{MoS}_{2}$ and $1 \mathrm{~L}-\mathrm{WSe} e_{2}$. Specifically, the change in $\mathrm{pH}$ results in a change of the redox potentials of the $\mathrm{O}_{2} / \mathrm{H}_{2} \mathrm{O}$ couple tested in this study (from -5.54 to $-4.95 \mathrm{eV}$ ) between $\mathrm{pH} 2$ and 12 , and the oxidation and reduction potentials for $1 \mathrm{~L}$ $\mathrm{WSe}_{2}$ are also shifted. ${ }^{42}$ When $\mathrm{H}^{+}$(or $\mathrm{OH}^{-}$) is directly involved in the oxidization/reduction reaction, as in eq 3 , the relative potentials between $\mathrm{O}_{2} / \mathrm{H}_{2} \mathrm{O}$ and $1 \mathrm{~L}-\mathrm{WSe} \mathrm{e}_{2}$ will change, ${ }^{42}$ becoming one of the factors influencing the $\mathrm{pH}$ dependent degradation effect.

Furthermore, $\mathrm{WO}_{3}$ will be involved in a more complicated $\mathrm{pH}$-dependent reaction in aqueous solution because of its thermodynamic instability. ${ }^{54,55}$ To further understand the $\mathrm{pH}$ dependence, we also consider the second reaction of the oxidation product

$$
\mathrm{WO}_{3}+\mathrm{H}_{2} \mathrm{O} \rightleftharpoons \mathrm{WO}_{4}{ }^{2-}+2 \mathrm{H}^{+}
$$

whose reaction rate also strongly depends on the solution $\mathrm{pH}$. According to eqs 3 and 4, the $\mathrm{pH}$ dependence of the photodegradation rate can be understood as follows: under the basic condition, the reaction resulting in the dissolution of $\mathrm{WO}_{3}$ should become faster than that under the acidic condition. Thus, the fast degradation under the basic condition is attributable to both the enhanced oxidation reaction and the dissolution reaction of $\mathrm{WSe}_{2}$ and $\mathrm{WO}_{3}$, which generate more defects and edges ${ }^{56-59}$ in the $1 \mathrm{~L}-\mathrm{WSe}_{2}$ flake. This hypothesis is supported by the $\mathrm{pH}$ dependence of the decrease of the PL intensities (Figure 3). For the PL intensity under the acidic condition, the time trace is well fitted by a single-exponential function. By contrast, the trace corresponding to the basic condition shows a much faster initial degradation that cannot be explained by a single reaction, implying involvement of an additional electrochemical process with a different time scale, as expressed in eq 4 . WSe $e_{2}$ degradation species differ among the acidic $\left(\mathrm{WO}_{3}\right.$ and $\left.\mathrm{SeO}_{4}{ }^{2-}\right)$, neutral, and basic conditions $\left(\mathrm{WO}_{4}{ }^{2-}\right.$ and $\left.\mathrm{SeO}_{4}{ }^{2-}\right){ }^{60}$ This observation is consistent with the recently reported $\mathrm{pH}$-dependent Gibbs free energy of $\mathrm{WSe}_{2}{ }^{60}$ For samples under acidic conditions, we can simply set the degradation rate equation as $\mathrm{d} A / \mathrm{d} t=-\alpha A$, where $A$ is the undegraded area of $1 \mathrm{~L}-\mathrm{WSe}_{2}$ at time $t$ and $\alpha$ is the rate constant. Solving this equation gives $A=A_{0} \mathrm{e}^{-\alpha t}$, where $A_{0}$ is the original area of $1 \mathrm{~L}-\mathrm{WSe} \mathrm{e}_{2}$. The undegraded area of $1 \mathrm{~L}-\mathrm{WSe}_{2}$ decreases exponentially, and the decrease in PL intensity can be well reproduced using a single-exponential function, as shown in Figure 3.

To further confirm the photoinduced degradation effects, we measured the PL properties of the samples kept in the dark and in degassed water. The samples kept under dark conditions were not illuminated except during the measurements. As shown in Figure 5, the degradation reactions of $1 \mathrm{~L}-\mathrm{WSe}_{2}$ in both dark (not degassed) ( 46\%) and degassed (with light) conditions $(\sim 38 \%)$ for $30 \mathrm{~min}$ are much slower than that of $1 \mathrm{~L}-\mathrm{WSe}_{2}$ in distilled water (not degassed, with light) ( $\left.\sim 82 \%\right)$, which supports our hypothesis that the degradation is mainly induced by the electrochemical effects of the photogenerated holes and dissolved oxygen in water. ${ }^{61,62}$

The effect of photogenerated carrier density on the degradation rate was also confirmed. As shown in Figure 6, 


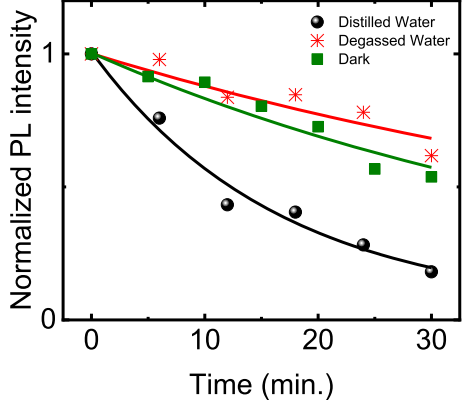

Figure 5. Time-dependent PL intensity of $1 \mathrm{~L}-\mathrm{WSe}_{2}$ in distilled water, water in the absence of light (dark) and degassed water. The solid curves are the results of fitting using single-exponential functions.

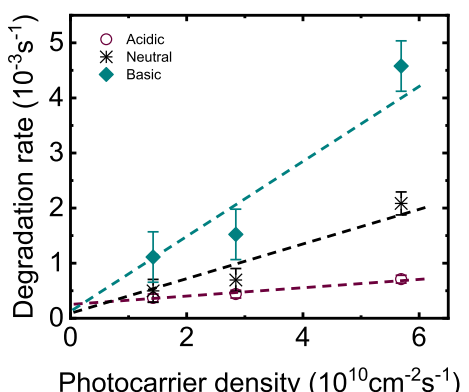

Figure 6. Photocarrier-density-dependent degradation rate in acidic, neutral, and basic conditions. The dashed lines are the results of fitting.

the degradation rates of $1 \mathrm{~L}-\mathrm{WS} e_{2}$ under all conditions (acidic, neutral, and basic) are nearly proportional to the density of photogenerated electrons/holes, which strongly supports the hypothesis that degradation occurs mainly from photoinduced effects. The wavelength-dependent degradation rate, as shown in Figure S4, further supports this hypothesis. Moreover, under the acidic condition, the degradation rate was almost one order of magnitude smaller than that under the basic condition, which also supports the aforementioned hypothesis. By extrapolation, the degradation rate is between $0.1 \times 10^{-3}$ and $0.3 \times 10^{-3} \mathrm{~s}^{-1}$ when the photocarrier density is 0 (Figure 6 ), consistent with the degradation rate in the dark (Figure 5), where a slow degradation with a rate of $\sim 0.3 \times 10^{-3} \mathrm{~s}^{-1}$ is observed.

\section{CONCLUSIONS}

In summary, we studied the stability of $1 \mathrm{~L}-\mathrm{WSe}_{2}$ and $1 \mathrm{~L}-\mathrm{MoS}_{2}$ in ambient air, distilled water, and acidic/basic aqueous solutions under light irradiation. In contrast to the good stability of both materials in ambient air, $1 \mathrm{~L}-\mathrm{MoS}_{2}$ was found to be much more stable than $1 \mathrm{~L}-\mathrm{WSe}_{2}$ under aqueous conditions. These results were consistently attributed to electrochemical reactions involving photoinduced electrons/ holes at the interface of $1 \mathrm{~L}-\mathrm{WSe}_{2}$ and the $\mathrm{O}_{2} / \mathrm{H}_{2} \mathrm{O}$ redox system. A much lower degradation rate was observed for $1 \mathrm{~L}$ $\mathrm{WSe}_{2}$ under acidic conditions than under basic conditions, which we attributed mainly to the $\mathrm{pH}$ dependence of the oxidation/reduction potential of $\mathrm{WSe}_{2}$ and to the further reaction of the oxidation species. We also confirmed that the degradation rates of $1 \mathrm{~L}-\mathrm{WSe}_{2}$ in both degassed water and dark conditions are much lower than those of $1 \mathrm{~L}-\mathrm{WSe}_{2}$ in distilled water. The slower degradation in degassed water and dark conditions supports our hypothesis that photoinduced electrons/holes and dissolved oxygen are the main contributors to the degradation of $1 \mathrm{~L}-\mathrm{WSe}_{2}$. Our results may provide insights into the effects of photogenerated electrons/holes and electrochemical phenomena at the interface of atomically thin semiconductors and an $\mathrm{O}_{2} / \mathrm{H}_{2} \mathrm{O}$ redox system on their stability, which is important for their applications in realistic environments because these effects are unavoidable when a monolayer material with a large specific surface area is in a practical environment with finite humidity or in solution.

\section{EXPERIMENTAL METHODS}

1L-TMDC samples were prepared from a bulk single crystal (hq graphene) using a standard mechanical exfoliation method. The thickness of an exfoliated TMDC was first determined via image contrast using an optical microscope, and the Raman and PL spectra were then used to confirm the thickness. The exfoliated 1L-TMDCs were transferred onto a transparent glass substrate using polydimethylsiloxane, and the substrate with $1 \mathrm{~L}-\mathrm{TMDCs}$ was then tightly bound with a glass tube to compose a liquid reservoir in which sufficient liquid could be kept over the sample for the optical measurements in aqueous solutions. $\mathrm{H}_{2} \mathrm{SO}_{4}$ and $\mathrm{NaOH}$ were used as the $\mathrm{pH}$ adjustors to attain acidic, neutral, or basic aqueous solutions. Degassed water was prepared using argon gas bubbling to reduce the dissolved oxygen concentration.

Optical measurements were performed through the transparent glass substrate using a homemade optical measurement system with a supercontinuum light source $(20$ ps pulse duration and $40 \mathrm{MHz}$ frequency) equipped with a monochromator (Figure 1). PL spectra were recorded with the samples in ambient air, in water, and finally in acidic and basic aqueous solutions using a liquid nitrogen-cooled silicon CCD detector. The measurements were performed at regular intervals $(5 \mathrm{~min})$ with a signal acquisition time of $5 \mathrm{~s}$. During the measurements, samples were kept illuminated by light with a power density of $800 \mathrm{~W} \mathrm{~cm}^{-2}$, which is in the linear region as shown in Figure S2, except for the experiment under dark conditions. The amount of water and aqueous solutions was kept the same, and all the measurements were performed at room temperature.

\section{ASSOCIATED CONTENT}

\section{Supporting Information}

The Supporting Information is available free of charge on the ACS Publications website at DOI: 10.1021/acsomega.9b01067.

Fundamental Raman and PL spectra of $1 \mathrm{~L}-\mathrm{WSe}_{2}$ and $1 \mathrm{~L}-\mathrm{MoS}_{2}$ used in this study; excitation power dependence of PL intensity of $1 \mathrm{~L}-\mathrm{WSe}_{2}$ and the wavelength dependence of the degradation rate in the distilled water condition; optical and PL images of $1 \mathrm{~L}-\mathrm{WSe}_{2}$ before and after photoirradiation (PDF)

\section{AUTHOR INFORMATION}

\section{Corresponding Author}

*E-mail: miyauchi@iae.kyoto-u.ac.jp.

ORCID $\odot$

Kazunari Matsuda: 0000-0002-3990-8484

Yuhei Miyauchi: 0000-0002-0945-0265

Notes

The authors declare no competing financial interest. 


\section{ACKNOWLEDGMENTS}

This research was supported by JSPS KAKENHI grant numbers JP16H00911, JP15K13337, JP15H05408, JP15K13500, JP16H00910, JP16H06331, JP17K19055, by the Asahi Glass Foundation, by JST CREST (JPMJCR16F3, JPMJCR18I5), by the Research Foundation for Opto-Science and Technology, and by the Nakatani Foundation.

\section{REFERENCES}

(1) Mak, K. F.; Lee, C.; Hone, J.; Shan, J.; Heinz, T. Atomically Thin $\mathrm{MoS}_{2}$ : A New Direct-Gap Semiconductor. Phys. Rev. Lett. 2010, 105, 136805.

(2) Yu, H.; Cui, X.; Xu, X.; Yao, W. Valley Excitons in TwoDimensional Semiconductors. Nat. Sci. Rev. 2015, 2, 57-70.

(3) Kang, J.; Zhang, L.; Wei, S.-H. A Unified Understanding of the Thickness-Dependent Bandgap Transition in Hexagonal Two-Dimensional Semiconductors. J. Phys. Chem. Lett. 2016, 7, 597-602.

(4) Nouchi, R.; Tanigaki, K. Charge-Density Depinning at Metal Contacts of Graphene Field-Effect Transistors. Appl. Phys. Lett. 2010, 96, 253503.

(5) Yin, Z.; Li, H.; Li, H.; Jiang, L.; Shi, Y.; Sun, Y.; Lu, G.; Zhang, Q.; Chen, X.; Zhang, H. Single-Layer $\mathrm{MoS}_{2}$ Phototransistors. ACS Nano 2012, 6, 74-80.

(6) Liu, W.; Kang, J.; Sarkar, D.; Khatami, Y.; Jena, D.; Banerjee, K. Role of Metal Contacts in Designing High-Performance Monolayer nType WSe ${ }_{2}$ Field Effect Transistors. Nano Lett. 2013, 13, 1983-1990.

(7) Wu, C.-C.; Jariwala, D.; Sangwan, V. K.; Marks, T. J.; Hersam, M. C.; Lauhon, L. J. Elucidating the Photoresponse of Ultrathin $\mathrm{MoS}_{2}$ Field-Effect Transistors by Scanning Photocurrent Microscopy. J. Phys. Chem. Lett. 2013, 4, 2508-2513.

(8) Gao, Y.; Liu, Z.; Sun, D.-M.; Huang, L.; Ma, L.-P.; Yin, L.-C.; Ma, T.; Zhang, Z.; Ma, X.-L.; Peng, L.-M.; et al. Large-Area Synthesis of High-Quality and Uniform Monolayer $\mathrm{WS}_{2}$ on Reusable Au Foils. Nat. Commun. 2015, 6, 8569.

(9) Kretschmer, S.; Komsa, H.-P.; Bøggild, P.; Krasheninnikov, A. V. Structural Transformations in Two-Dimensional Transition-Metal Dichalcogenide $\mathrm{MoS}_{2}$ Under Electron Beam: Insights from FirstPrinciples Calculations. J. Phys. Chem. Lett. 2017, 8, 3061-3067.

(10) Lopez-Sanchez, O.; Lembke, D.; Kayci, M.; Radenovic, A.; Kis, A. Ultrasensitive Photodetectors Based on Monolayer $\mathrm{MoS}_{2}$. Nat. Nanotechnol. 2013, 8, 497-501.

(11) Tan, D.; Lim, H. E.; Wang, F.; Mohamed, N. B.; Mouri, S.; Zhang, W.; Miyauchi, Y.; Ohfuchi, M.; Matsuda, K. Anisotropic Optical and Electronic Properties of Two-Dimensional Layered Germanium Sulfide. Nano Res. 2017, 10, 546-555.

(12) Yu, W.; Li, S.; Zhang, Y.; Ma, W.; Sun, T.; Yuan, J.; Fu, K.; Bao, Q. Near-Infrared Photodetectors Based on $\mathrm{MoTe}_{2} /$ Graphene Heterostructure with High Responsivity and Flexibility. Small 2017, 13, 1700268.

(13) Sundaram, R. S.; Engel, M.; Lombardo, A.; Krupke, R.; Ferrari, A. C.; Avouris, P.; Steiner, M. Electroluminescence in Single Layer $\mathrm{MoS}_{2}$. Nano Lett. 2013, 13, 1416-1421.

(14) Ross, J. S.; Klement, P.; Jones, A. M.; Ghimire, N. J.; Yan, J.; Mandrus, D. G.; Taniguchi, T.; Watanabe, K.; Kitamura, K.; Yao, W.; et al. Electrically Tunable Excitonic Light-Emitting Diodes Based on Monolayer $\mathrm{WSe}_{2}$ p-n Junctions. Nat. Nanotechnol. 2014, 9, 268-272.

(15) Tongay, S.; Zhou, J.; Ataca, C.; Liu, J.; Kang, J. S.; Matthews, T. S.; You, L.; Li, J.; Grossman, J. C.; Wu, J. Broad-Range Modulation of Light Emission in Two-Dimensional Semiconductors by Molecular Physisorption Gating. Nano Lett. 2013, 13, 2831-2836.

(16) Bernardi, M.; Palummo, M.; Grossman, J. C. Extraordinary Sunlight Absorption and One Nanometer Thick Photovoltaics Using Two-Dimensional Monolayer Materials. Nano Lett. 2013, 13, 36643670.

(17) Tsuboi, Y.; Wang, F.; Kozawa, D.; Funahashi, K.; Mouri, S.; Miyauchi, Y.; Takenobu, T.; Matsuda, K. Enhanced Photovoltaic Performances of Graphene/Si Solar Cells by Insertion of a $\mathrm{MoS}_{2}$ Thin Film. Nanoscale 2015, 7, 14476-14482.
(18) Zhou, Y.; Xu, W.; Sheng, Y.; Huang, H.; Zhang, Q.; Hou, L.; Shautsova, V.; Warner, J. H. Symmetry-Controlled Reversible Photovoltaic Current Flow in Ultrathin All 2D Vertically Stacked Graphene $/ \mathrm{MoS}_{2} / \mathrm{WS}_{2} /$ Graphene Devices. ACS Appl. Mater. Interfaces 2019, 11, 2234-2242.

(19) Mouri, S.; Miyauchi, Y.; Matsuda, K. Tunable Photoluminescence of Monolayer $\mathrm{MoS}_{2}$ via Chemical Doping. Nano Lett. 2013, 13, 5944-5948.

(20) Dhakal, K. P.; Duong, D. L.; Lee, J.; Nam, H.; Kim, M.; Kan, M.; Lee, Y. H.; Kim, J. Confocal Absorption Spectral Imaging of $\mathrm{MoS}_{2}$ : Optical Transitions Depending on the Atomic Thickness of Intrinsic and Chemically Doped $\mathrm{MoS}_{2}$. Nanoscale 2014, 6, 1302813035 .

(21) Kiriya, D.; Tosun, M.; Zhao, P.; Kang, J. S.; Javey, A. Air-Stable Surface Charge Transfer Doping of $\mathrm{MoS}_{2}$ by Benzyl Viologen. J. Am. Chem. Soc. 2014, 136, 7853-7856.

(22) Wang, S.; Zhao, W.; Giustiniano, F.; Eda, G. Effect of Oxygen and Ozone on P-Type Doping of Ultra-Thin $\mathrm{WSe}_{2}$ and $\mathrm{MoSe}_{2}$ Field Effect Transistors. Phys. Chem. Chem. Phys. 2016, 18, 4304-4309.

(23) Suh, J.; Park, T.-E.; Lin, D.-Y.; Fu, D.; Park, J.; Jung, H. J.; Chen, Y.; Ko, C.; Jang, C.; Sun, Y.; et al. Doping against the Native Propensity of $\mathrm{MoS}_{2}$ : Degenerate Hole Doping by Cation Substitution. Nano Lett. 2014, 14, 6976-6982.

(24) Nan, H.; Wang, Z.; Wang, W.; Liang, Z.; Lu, Y.; Chen, Q.; He, D.; Tan, P.; Miao, F.; Wang, X.; et al. Strong Photoluminescence Enhancement of $\mathrm{MoS}_{2}$ through Defect Engineering and Oxygen Bonding. ACS Nano 2014, 8, 5738-5745.

(25) Mak, K. F.; He, K.; Lee, C.; Lee, G. H.; Hone, J.; Heinz, T. F.; Shan, J. Tightly Bound Trions in Monolayer $\mathrm{MoS}_{2}$. Nat. Mater. 2013, 12, 207-211.

(26) Atallah, T. L.; Wang, J.; Bosch, M.; Seo, D.; Burke, R. A.; Moneer, O.; Zhu, J.; Theibault, M.; Brus, L. E.; Hone, J.; et al. Electrostatic Screening of Charged Defects in Monolayer $\mathrm{MoS}_{2}$. J. Phys. Chem. Lett. 2017, 8, 2148-2152.

(27) Liu, B.; Chen, L.; Liu, G.; Abbas, A. N.; Fathi, M.; Zhou, C. High-Performance Chemical Sensing Using Schottky-Contacted Chemical Vapor Deposition Grown Monolayer $\mathrm{MoS}_{2}$ Transistors. ACS Nano 2014, 8, 5304-5314.

(28) Bertolazzi, S.; Bonacchi, S.; Nan, G.; Pershin, A.; Beljonne, D.; Samorì, P. Engineering Chemically Active Defects in Monolayer $\mathrm{MoS}_{2}$ Transistors via Ion-Beam Irradiation and Their Healing via Vapor Deposition of Alkanethiols. Adv. Mater. 2017, 29, 1606760.

(29) Neupane, G. P.; Tran, M. D.; Yun, S. J.; Kim, H.; Seo, C.; Lee, J.; Han, G. H.; Sood, A. K.; Kim, J. Simple Chemical Treatment to NDope Transition-Metal Dichalcogenides and Enhance the Optical and Electrical Characteristics. ACS Appl. Mater. Interfaces 2017, 9, 1195011958.

(30) Voiry, D.; Yamaguchi, H.; Li, J.; Silva, R.; Alves, D. C. B.; Fujita, T.; Chen, M.; Asefa, T.; Shenoy, V. B.; Eda, G.; et al. Enhanced Catalytic Activity in Strained Chemically Exfoliated $\mathrm{WS}_{2}$ Nanosheets for Hydrogen Evolution. Nat. Mater. 2013, 12, 850-855.

(31) Zhuang, H. L.; Hennig, R. G. Single-Layer Group-III Monochalcogenide Photocatalysts for Water Splitting. Chem. Mater. 2013, 25, 3232-3238.

(32) Late, D. J.; Huang, Y.-K.; Liu, B.; Acharya, J.; Shirodkar, S. N.; Luo, J.; Yan, A.; Charles, D.; Waghmare, U. V.; Dravid, V. P.; et al. Sensing Behavior of Atomically Thin-Layered $\mathrm{MoS}_{2}$ Transistors. ACS Nano 2013, 7, 4879-4891.

(33) Lee, J.; Dak, P.; Lee, Y.; Park, H.; Choi, W.; Alam, M. A.; Kim, S. Two-Dimensional Layered $\mathrm{MoS}_{2}$ Biosensors Enable Highly Sensitive Detection of Biomolecules. Sci. Rep. 2014, 4, 7352.

(34) Rao, C. N. R.; Gopalakrishnan, K.; Maitra, U. Comparative Study of Potential Applications of Graphene, $\mathrm{MoS}_{2}$, and Other TwoDimensional Materials in Energy Devices, Sensors, and Related Areas. ACS Appl. Mater. Interfaces 2015, 7, 7809-7832.

(35) Ko, K. Y.; Park, K.; Lee, S.; Kim, Y.; Woo, W. J.; Kim, D.; Song, J.-G.; Park, J.; Kim, H. Recovery Improvement for Large-Area Tungsten Diselenide Gas Sensors. ACS Appl. Mater. Interfaces 2018, 10, 23910-23917. 
(36) Sunamura, K.; Page, T. R.; Yoshida, K.; Yano, T.-a.; Hayamizu, Y. Laser-Induced Electrochemical Thinning of $\mathrm{MoS}_{2}$. J. Mater. Chem. C 2016, 4, 3268-3273.

(37) Oh, H. M.; Han, G. H.; Kim, H.; Bae, J. J.; Jeong, M. S.; Lee, Y. $\mathrm{H}$. Photochemical Reaction in Monolayer $\mathrm{MoS}_{2}$ via Correlated Photoluminescence, Raman Spectroscopy, and Atomic Force Microscopy. ACS Nano 2016, 10, 5230-5236.

(38) Yamamoto, M.; Dutta, S.; Aikawa, S.; Nakaharai, S.; Wakabayashi, K.; Fuhrer, M. S.; Ueno, K.; Tsukagoshi, K. SelfLimiting Layer-by-Layer Oxidation of Atomically Thin $\mathrm{WSe}_{2}$. Nano Lett. 2015, 15, 2067-2073.

(39) Liu, H.-L.; Shen, C.-C.; Su, S.-H.; Hsu, C.-L.; Li, M.-Y.; Li, L.-J. Optical Properties of Monolayer Transition Metal Dichalcogenides Probed by Spectroscopic Ellipsometry. Appl. Phys. Lett. 2014, 105, 201905.

(40) Arora, A.; Koperski, M.; Nogajewski, K.; Marcus, J.; Faugeras, C.; Potemski, M. Excitonic Resonances in Thin Films of $\mathrm{WSe}_{2}$ : From Monolayer to Bulk Material. Nanoscale 2015, 7, 10421-10429.

(41) Zhang, W.; Matsuda, K.; Miyauchi, Y. PH-Dependent Photoluminescence Properties of Monolayer Transition-Metal Dichalcogenides Immersed in an Aqueous Solution. J. Phys. Chem. C 2018, 122, 13175-13181.

(42) Chen, S.; Wang, L.-W. Thermodynamic Oxidation and Reduction Potentials of Photocatalytic Semiconductors in Aqueous Solution. Chem. Mater. 2012, 24, 3659-3666.

(43) Chakrapani, V.; Angus, J. C.; Anderson, A. B.; Wolter, S. D.; Stoner, B. R.; Sumanasekera, G. U. Charge Transfer Equilibria Between Diamond and an Aqueous Oxygen Electrochemical Redox Couple. Science 2007, 318, 1424-1430.

(44) Aguirre, C. M.; Levesque, P. L.; Paillet, M.; Lapointe, F.; StAntoine, B. C.; Desjardins, P.; Martel, R. The Role of the Oxygen/ Water Redox Couple in Suppressing Electron Conduction in FieldEffect Transistors. Adv. Mater. 2009, 21, 3087-3091.

(45) Wang, F.; Shifa, T. A.; Zhan, X.; Huang, Y.; Liu, K.; Cheng, Z.; Jiang, C.; He, J. Recent Advances in Transition-Metal Dichalcogenide Based Nanomaterials for Water Splitting. Nanoscale 2015, 7, 1976419788 .

(46) Liu, Y.; Stradins, P.; Wei, S.-H. Van Der Waals MetalSemiconductor Junction: Weak Fermi Level Pinning Enables Effective Tuning of Schottky Barrier. Sci. Adv. 2016, 2, No. e1600069.

(47) Zhang, C.; Gong, C.; Nie, Y.; Min, K.-A.; Liang, C.; Oh, Y. J.; Zhang, H.; Wang, W.; Hong, S.; Colombo, L.; et al. Systematic Study of Electronic Structure and Band Alignment of Monolayer Transition Metal Dichalcogenides in Van Der Waals Heterostructures. 2D Mater. 2016, 4, 015026.

(48) Choudhury, P.; Ravavarapu, L.; Dekle, R.; Chowdhury, S. Modulating Electronic and Optical Properties of Monolayer $\mathrm{MoS}_{2}$ Using Nonbonded Phthalocyanine Molecules. J. Phys. Chem. C 2017, 121, 2959-2967.

(49) Jaramillo, T. F.; Jørgensen, K. P.; Bonde, J.; Nielsen, J. H.; Horch, S.; Chorkendorff, I. Identification of Active Edge Sites for Electrochemical $\mathrm{H}_{2}$ Evolution from $\mathrm{MoS}_{2}$ Nanocatalysts. Science 2007, 317, 100-102.

(50) Joshi, R. K.; Shukla, S.; Saxena, S.; Lee, G.-H.; Sahajwalla, V.; Alwarappan, S. Hydrogen Generation via Photoelectrochemical Water Splitting Using Chemically Exfoliated $\mathrm{MoS}_{2}$ Layers. AIP $A d v$ 2016, 6, 015315 .

(51) Rasmussen, F. A.; Thygesen, K. S. Computational 2D Materials Database: Electronic Structure of Transition-Metal Dichalcogenides and Oxides. J. Phys. Chem. C 2015, 119, 13169-13183.

(52) Fujii, K. In Photoelectrochemical Solar Fuel Production, 1st ed.; Giménez, S., Bisquert, J., Eds.; Springer International Publishing, 2016.

(53) Böhmisch, M.; Burmeister, F.; Boneberg, J.; Leiderer, P. Nanostructuring on $\mathrm{WSe}_{2}$ with the Atomic Force Microscope by a Potential Controlled Electrochemical Reaction. Appl. Phys. Lett. 1996, 69, 1882-1884.

(54) Huang, Y.-T.; Dodda, A.; Schulman, D. S.; Sebastian, A.; Zhang, F.; Buzzell, D.; Terrones, M.; Feng, S.-P.; Das, S. Anomalous
Corrosion of Bulk Transition Metal Diselenides Leading to Stable Monolayers. ACS Appl. Mater. Interfaces 2017, 9, 39059-39068.

(55) Eng, A. Y. S.; Ambrosi, A.; Sofer, Z.; Simek, P.; Pumera, M. Electrochemistry of Transition Metal Dichalcogenides: Strong Dependence on the Metal-to-Chalcogen Composition and Exfoliation Method. ACS Nano 2014, 8, 12185-12198.

(56) Liu, X.; Xu, T.; Wu, X.; Zhang, Z.; Yu, J.; Qiu, H.; Hong, J. H.; Jin, C. H.; Li, J. X.; Wang, X. R.; et al. Top-down Fabrication of SubNanometre Semiconducting Nanoribbons Derived from Molybdenum Disulfide Sheets. Nat. Commun. 2013, 4, 1776.

(57) Nozaki, J.; Fukumura, M.; Aoki, T.; Maniwa, Y.; Yomogida, Y.; Yanagi, K. Manipulation of Local Optical Properties and Structures in Molybdenum-Disulfide Monolayers Using Electric Field-Assisted near-Field Techniques. Sci. Rep. 2017, 7, 46004.

(58) Ryu, Y.; Kim, W.; Koo, S.; Kang, H.; Watanabe, K.; Taniguchi, T.; Ryu, S. Interface-Confined Doubly Anisotropic Oxidation of TwoDimensional $\mathrm{MoS}_{2}$. Nano Lett. 2017, 17, 7267-7273.

(59) Das, S. R.; Wakabayashi, K.; Yamamoto, M.; Tsukagoshi, K.; Dutta, S. Layer-by-Layer Oxidation Induced Electronic Properties in Transition-Metal Dichalcogenides. J. Phys. Chem. C 2018, 122, 17001-17007.

(60) Singh, A. K.; Zhou, L.; Shinde, A.; Suram, S. K.; Montoya, J. H.; Winston, D.; Gregoire, J. M.; Persson, K. A. Electrochemical Stability of Metastable Materials. Chem. Mater. 2017, 29, 10159-10167.

(61) Yin, M.; Li, Z.; Kou, J.; Zou, Z. Mechanism Investigation of Visible Light-Induced Degradation in a Heterogeneous $\mathrm{TiO}_{2} /$ Eosin Y/Rhodamine B System. Environ. Sci. Technol. 2009, 43, 8361-8366.

(62) Gao, J.; Li, B.; Tan, J.; Chow, P.; Lu, T.-M.; Koratkar, N. Aging of Transition Metal Dichalcogenide Monolayers. ACS Nano 2016, 10, $2628-2635$. 